\title{
Research Article \\ Finite-Horizon Optimal Control of Discrete-Time Switched Linear Systems
}

\author{
Qixin Zhu' ${ }^{1,2}$ and Guangming $X i e^{1,2}$ \\ ${ }^{1}$ The Center for System and Control, College of Engineering, Peking University, Beijing 100871, China \\ ${ }^{2}$ School of Electrical and Electronic Engineering, East China Jiaotong University, Nanchang 330013, China \\ ${ }^{3}$ Department of Mechanical and Electrical Engineering, Suzhou University of Science and Technology, \\ Suzhou 215011, China
}

Correspondence should be addressed to Qixin Zhu, bob21cn@163.com

Received 1 May 2012; Revised 2 July 2012; Accepted 10 July 2012

Academic Editor: Soohee Han

Copyright (C) 2012 Q. Zhu and G. Xie. This is an open access article distributed under the Creative Commons Attribution License, which permits unrestricted use, distribution, and reproduction in any medium, provided the original work is properly cited.

\begin{abstract}
Finite-horizon optimal control problems for discrete-time switched linear control systems are investigated in this paper. Two kinds of quadratic cost functions are considered. The weight matrices are different. One is subsystem dependent; the other is time dependent. For a switched linear control system, not only the control input but also the switching signals are control factors and are needed to be designed in order to minimize cost function. As a result, optimal design for switched linear control systems is more complicated than that of non-switched ones. By using the principle of dynamic programming, the optimal control laws including both the optimal switching signal and the optimal control inputs are obtained for the two problems. Two examples are given to verify the theory results in this paper.
\end{abstract}

\section{Introduction}

A switched system usually consists of a family of subsystems described by differential or difference equations and a logical rule that dominates the switching among them. Such systems arise in many engineering fields, such as power electronics, embedded systems, manufacturing, and communication networks. In the past decade or so, the analysis and synthesis of switched linear control systems have been extensively studied [1-28]. Compared with the traditional optimal control problems, not only the control input but also the switching signals needed to be designed to minimize the cost function.

The first focus of this paper is on the finite-horizon optimal regulation for discretetime switched linear systems. The goal of this paper is to develop a set of optimal control strategies that minimizes the given quadratic cost function. The problem is of fundamental 
importance in both theory and practice and has challenged researchers for many years. The bottleneck is mostly on the determination of the optimal switching strategy. Many methods have been proposed to tackle this problem. Algorithms for optimizing the switching instants with a fixed mode sequence have been derived for general switched systems in [29] and for autonomous switched systems in [30].

The finite-horizon optimal control problems for discrete-time switched linear control systems are investigated in [31]. Motivated by this work, two kinds of quadratic cost functions are considered in this paper. The former is introduced in [31], where the state and input weight matrices are subsystem dependent. We form the later by ourselves, where the weight matrices are time dependent. According to these two kinds of cost functions, we formulate two finite-horizon optimal control problems. As a result, two novel Riccati mappings are built up. They are equivalent to that in [31]. Actually, the optimal quadratic regulation for discrete-time switched linear systems has been discussed in [31]. However, there are at least one difference between this paper and [31]. That is to say the control strategies proposed in this paper are not the same as that of [31].

This paper is organized into six sections including the introduction. Section 2 presents the problem formulation. Section 3 presents the optimal control of discrete-time switched linear system. Two examples are given in Section 4. Section 5 summaries this paper.

Notations. Notations in this paper are quite standard. The superscript " $T$ " stands for the transpose of a matrix. $R^{n}$ and $R^{n \times m}$ denote the $n$ dimensional Euclidean space and the set of all $n \times m$ real matrices, respectively. The notation $X>0(X \geq 0)$ means the matrix $X$ is positive definite ( $X$ is semipositive definite).

\section{Problem Formulation}

Consider the discrete-time switched linear system defined as

$$
x(k+1)=A_{r(k)} x(k)+B_{r(k)} u(k), \quad k=0,1, \ldots N-1,
$$

where $x(k) \in R^{n}$ is the state, $u(k) \in R^{p}$ is the control input, and $r(k) \in M=\{1,2, \ldots, d\}$ is the switching signal to be designed. For each $i \in M, A_{i}$ and $B_{i}$ are constant matrices of appropriate dimension, and the pair $\left(A_{i}, B_{i}\right)$ is called a subsystem of (2.1). This switched linear system is time invariant in the sense that the set of available subsystems $\left\{\left(A_{i}, B_{i}\right)\right\}_{i=1}^{d}$ is independent of time $k$. We assume that there is no internal forced switching, that is, the system can stay at or switch to any mode at any time instant. It is assumed that the initial state of the system $x(0)=x_{0}$ is a constant.

Due to the switching signal, different from the traditional optimal control problem for linear time-invariant systems, two kinds of cost function for finite-horizon optimal control of discrete-time switched linear systems are introduced. The first one is

$$
J_{1}(u, r)=x(N)^{T} Q_{f} x(N)+\sum_{j=0}^{N-1}\left[x(j)^{T} Q_{r(j)} x(j)+u(j)^{T} R_{r(j)} u(j)\right]
$$

where $Q_{f}=Q_{f}^{T} \geq 0$ is the terminal state weight matrix, $Q_{i}=Q_{i}^{T}>0$ and $R_{i}=R_{i}^{T}>0$ are running weight matrices for the state and the input for subsystem $i \in M$. 
The second one is

$$
J_{2}(u, r)=x(N)^{T} Q_{f} x(N)+\sum_{j=0}^{N-1}\left[x(j)^{T} Q_{j} x(j)+u(j)^{T} R_{j} u(j)\right]
$$

where $Q_{f}=Q_{f}^{T} \geq 0$ is the terminal state weight matrix, $Q_{i}=Q_{i}^{T}>0$ and $R_{i}=R_{i}^{T}>0$ are running weight matrices for the state and the input at the time instant $j \in\{0,1, \ldots, N-1\}$.

Remark 2.1. The cost function $J_{1}$, is introduced in [31]. In $J_{1}$ the weight matrices are subsystem dependent. The cost function $J_{2}$ is introduced by us. In this case, the weight matrices are time dependent.

The goal of this paper is to solve the following two finite-horizon optimal control problems for switched linear systems.

Problem 1. Find the $u(j)$ and $r(j)$ that minimize $J_{1}(u, r)$ subject to the system (2.1).

Problem 2. Find the $u(j)$ and $r(j)$ that minimize $J_{2}(u, r)$ subject to the system (2.1).

\section{Optimal Solutions}

\subsection{Solutions to Problem 1}

To drive the minimum value of the cost function $J_{1}$ subject to system (2.1), we define the Riccati mapping $f_{i}: Y \rightarrow Y$ for each subsystem $\left(A_{i}, B_{i}\right)$ and weight matrices $Q_{i}$ and $R_{i}$, $i \in M$

$$
f_{i}(P)=\left(A_{i}-B_{i} K_{i}(P)\right)^{T} P\left(A_{i}-B_{i} K_{i}(P)\right)+K_{i}^{T}(P) R_{i} K_{i}(P)+Q_{i}
$$

where

$$
K_{i}(P)=\left(R_{i}+B_{i}^{T} P B_{i}\right)^{-1} B_{i}^{T} P A_{i}
$$

Let $H_{N}=\left\{Q_{f}\right\}$ be a set consisting of only one matrix $Q_{f}$. Define the set $H_{k}$ for $0 \leq k<N$ iteratively as

$$
H_{k}=\left\{X \mid X=f_{i}(P), \forall i \in M, P \in H_{k+1}\right\}
$$

Now we give the main result of this paper.

Theorem 3.1. The minimum value of the cost function $J_{1}$ in Problem 1 is

$$
J_{1}^{*}(u, r)=\min _{P \in H_{0}} x_{0}^{T} P x_{0}
$$


Furthermore, for $k \geq 0$, if one defines

$$
\left(P_{k}^{*}, i_{k}^{*}\right)=\arg \min _{P \in H_{k}} x(k)^{T} P x(k)
$$

then the optimal switching signal and the optimal control input at time instant $k$ are

$$
\begin{gathered}
r^{*}(k)=i_{k^{\prime}}^{*} \\
u^{*}(k)=-K_{i_{k}^{*}}\left(P_{k}^{*}\right) x(k),
\end{gathered}
$$

where $K_{i_{k}^{*}}\left(P_{k}^{*}\right)$ is defined by (3.2).

Proof. For the cost function $J_{1}$, by applying the principle of dynamic programming, we obtain the following Bellman equation when $k=0,1, \ldots, N-1$ :

$$
J_{1, k}(u, r)=\min _{i \in M, u \in R^{p}}\left\{x^{T}(k) Q_{i} x(k)+u^{T}(k) R_{i} u(k)+J_{1, k+1}(u, r)\right\}
$$

and the terminal condition

$$
J_{1, N}=x^{T}(N) Q_{s} x(N) .
$$

Now we will prove that the solution of the Bellman equation (3.8) and (3.9) may be written as

$$
J_{1, k}=\min _{P \in H_{k}} x^{T}(k) P x(k)
$$

We use mathematical induction to prove that (3.10) holds for $k=0,1, \ldots, N$.

(i) It is easy to see that (3.10) holds for $N$.

(ii) We assume that (3.10) holds for $k+1$, that is,

$$
J_{1, k+1}=\min _{P \in H_{k+1}} x^{T}(k+1) P x(k+1) .
$$


By (3.8), we have

$$
\begin{aligned}
J_{1, k}(u, r)= & \min _{i \in M, u(k) \in R^{p}}\left\{x^{T}(k) Q_{i} x(k)+u^{T}(k) R_{i} u(k)+\min _{P \in H_{k+1}} x^{T}(k+1) P x(k+1)\right\} \\
= & \min _{i \in M, u(k) \in R^{p}}\left\{x^{T}(k) Q_{i} x(k)+u^{T}(k) R_{i} u(k)+x^{T}(k+1) P_{k+1}^{*} x(k+1)\right\} \\
= & \min _{i \in M, u(k) \in R^{p}}\left\{x^{T}(k) Q_{i} x(k)+u^{T}(k) R_{i} u(k)\right. \\
+ & {\left.\left[A_{i} x(k)+B_{i} u(k)\right]^{T} P_{k+1}^{*}\left[A_{i} x(k)+B_{i} u(k)\right]\right\} } \\
= & \min _{i \in M, u(k) \in R^{p}}\left\{x^{T}(k)\left[Q_{i}+A_{i}^{T} P_{k+1}^{*} A_{i}\right] x(k)+u^{T}(k)\left[R_{i}+B_{i}^{T} P_{k+1}^{*} B_{i}\right] u(k)\right. \\
& \left.+2 x^{T}(k) A_{i}^{T} P_{k+1}^{*} B_{i} u(k)\right\} .
\end{aligned}
$$

Let

$$
H_{i}(u)=u^{T}\left(R_{i}+B_{i}^{T} P_{k+1}^{*} B_{i}\right) u+2 x^{T}(k) A_{i}^{T} P_{k+1}^{*} B_{i} u .
$$

By simple calculation, we have

$$
\frac{\partial H_{i}(u)}{\partial u}=2\left(R_{i}+B_{i}^{T} P_{k+1}^{*} B_{i}\right) u+2 B_{i}^{T} P_{k+1}^{*} A_{i} x(k)
$$

Since $u(k)$ is unconstrained, its optimal value $u_{i}^{*}(k)$ must satisfy $\partial H_{i}(u) / \partial u=0$.

It follows that

$$
u_{i}^{*}(k)=-\left(R_{i}+B_{i}^{T} P_{k+1}^{*} B_{i}\right)^{-1} B_{i}^{T} P_{k+1}^{*} A_{i} x(k)=-K_{i}\left(P_{k+1}^{*}\right) x(k)
$$

It follows that

$$
\begin{aligned}
J_{1, k}= & \min _{i \in M, u(k) \in R^{p}}\left\{x^{T}(k)\left[Q_{i}+A_{i}^{T} P_{k+1}^{*} A_{i}\right] x(k)+u_{i}^{* T}(k)\left[R_{i}+B_{i}^{T} P_{k+1}^{*} B_{i}\right] u_{i}^{*}(k)\right. \\
& \left.+2 x^{T}(k) A_{i}^{T} P_{k+1}^{*} B_{i} u_{i}^{*}(k)\right\} \\
= & \min _{i \in M}\left\{x^{T}(k)\left[Q_{i}+A_{i}^{T} P_{k+1}^{*} A_{i}\right] x(k)+x^{T}(k) K_{i}^{T}\left(P_{k+1}^{*}\right)\left[R_{i}+B_{i}^{T} P_{k+1}^{*} B_{i}\right] K_{i}\left(P_{k+1}^{*}\right) x(k)\right. \\
& \left.\quad-2 x^{T}(k) A_{i}^{T} P_{k+1}^{*} B_{i} K_{i}\left(P_{k+1}^{*}\right) x(k)\right\} \\
= & \min _{i \in M, P \in H_{k+1}} x^{T}(k) f_{i}(P) x(k) \\
= & \min _{P \in H_{k}} x^{T}(k) P x(k) .
\end{aligned}
$$


Then the optimal switching signal and the optimal control input at time $k$ are $\gamma^{*}(k)=i_{k}^{*}$ and $u^{*}(k)=-K_{i_{k}^{*}}\left(P_{k}^{*}\right) x(k)$, respectively. It means that (3.10) still holds for $k$. This completes the proof.

Remark 3.2. In [31], the optimal control input at time $k$ is $u^{*}(k)=-K_{i_{k}^{*}}\left(P_{k}^{*}\right) x(0)$, which is different with our result in (3.7).

Remark 3.3. In [31], another Riccati mapping is given by

$$
f_{i}(P)=Q_{i}+A_{i}^{T} P A_{i}-A_{i}^{T} P B_{i}\left(R_{i}+B_{i}^{T} P B_{i}\right)^{-1} B_{i}^{T} P A_{i}
$$

It is easy to verify that (3.17) and (3.1) are equivalent to each other. It should be strengthen that there is a matrix inverse operation in (3.17), while, in (3.1) is not. Thus, our result is more convenient for real application.

Remark 3.4. When $M=\{1\}$, the switched system (2.1) becomes a constant linear system $\left(A_{1}, B_{1}\right)=(A, B)$. In this case, the cost function $J_{1}$ becomes

$$
J_{1}(u)=x^{T}(N) Q_{f} x(N)+\sum_{j=0}^{N-1}\left[x^{T}(j) Q x(j)+u^{T}(j) R u(j)\right] .
$$

The Riccati mapping reduces to a discrete-time Riccati equation

$$
P_{k}^{*}=\left(A-B K_{k}^{*}\right)^{T} P_{k+1}^{*}\left(A-B K_{k}^{*}\right)+\left(K_{k}^{*}\right)^{T} R K_{k}^{*}+Q
$$

where

$$
K_{k}^{*}=\left(R+B^{T} P_{k+1}^{*} B\right)^{-1} B^{T} P_{k+1}^{*} A .
$$

It is easy to verify that this novel discrete-time Riccati equation (3.20) is also equivalent to the traditional ones, such as

$$
\begin{gathered}
P_{k}^{*}=Q+A^{T} P_{k+1}^{*} A-A^{T} P_{k+1}^{*} B\left(R+B^{T} P_{k+1}^{*} B\right)^{-1} B^{T} P_{k+1}^{*} A, \\
P_{k}^{*}=Q+A^{T}\left(\left(P_{k+1}^{*}\right)^{-1}+B^{T} R^{-1} B\right)^{-1} A, \\
P_{k}^{*}=Q+A^{T} P_{k+1}^{*} A\left(I+B^{T} R^{-1} P_{k+1}^{*}\right)^{-1} A .
\end{gathered}
$$




\subsection{Solutions to Problem 2}

To drive the minimum value of the cost function $J_{2}$ subject to system (2.1), we define the Riccati mapping $f_{i, k}: P \rightarrow P$ for each subsystem $\left(A_{i}, B_{i}\right)$ and weight matrices $Q_{k}$ and $R_{k}$, $i \in M, k=0,1, \ldots, N-1$ :

$$
f_{i, k}(P)=\left(A_{i}-B_{i} K_{i}(P)\right)^{T} P\left(A_{i}-B_{i} K_{i}(P)\right)+K_{i}^{T}(P) R_{k} K_{i}(P)+Q_{k}
$$

where

$$
K_{i}(P)=\left(R_{k}+B_{i}^{T} P B_{i}\right)^{-1} B_{i}^{T} P A_{i} .
$$

Let $J_{N}=\left\{Q_{f}\right\}$ be a set consisting of only one matrix $Q_{f}$. Define the set $L_{k}$ for $0 \leq k \leq N$ iteratively as

$$
L_{k}=\left\{X \mid X=f_{i, k}(P), \forall i \in M, P \in L_{k+1}\right\} \text {. }
$$

Then we give the following theorem.

Theorem 3.5. The minimum value of the cost function $\mathrm{J}_{2}$ in Problem 2 is

$$
J_{2}^{*}(u, r)=\min _{P \in L_{0}} x_{0}^{T} P x_{0} .
$$

Furthermore, for $k \geq 0$, if one defines

$$
\left(P_{k}^{*}, i_{k}^{*}\right)=\arg \min _{P \in L_{k}} x(k)^{T} P x(k),
$$

then the optimal switching signal and the optimal control input at time instant $k$ are

$$
\begin{gathered}
r^{*}(k)=i_{k^{\prime}}^{*} \\
u^{*}(k)=-K_{i_{k}^{*}}\left(P_{k}^{*}\right) x(k),
\end{gathered}
$$

where $K_{i_{k}^{*}}\left(P_{k}^{*}\right)$ is defined by (3.23).

The proof is similar to that of Theorem 3.1.

Proof. For the cost function $J_{2}$, by applying the principle of dynamic programming, we obtain the following Bellman equation:

$$
J_{2, k}(u, r)=\min _{i \in M, u \in R^{p}}\left\{x^{T}(k) Q_{k} x(k)+u^{T}(k) R_{k} u(k)+J_{2, k+1}(u, r)\right\}, \quad k=0,1, \ldots, N-1
$$

and the terminal condition

$$
J_{2, N}=x^{T}(N) Q_{s} x(N) .
$$


Now we will prove that the solution of the Bellman equation (3.29) (3.30) may be written as

$$
J_{2, k}(u, r)=\min _{P \in L_{k}} x^{T}(k) P x(k)
$$

We use mathematical induction to prove that (3.31) holds for $k=0,1, \ldots, N$.

(i) It is easy to verify (3.31) holds for $k=N$.

(ii) We assume (3.31) holds for $k+1$, that is,

$$
J_{1, k+1}(u, r)=\min _{P \in L_{k+1}} x^{T}(k+1) P x(k+1) .
$$

By (3.29), we have

$$
\begin{aligned}
J_{2, k}(u, r)= & \min _{i \in M, u(k) \in R^{p}}\left\{x^{T}(k) Q_{i} x(k)+u^{T}(k) R_{i} u(k)+\min _{P \in L_{k+1}} x^{T}(k+1) P x(k+1)\right\} \\
= & \min _{i \in M, u(k) \in R^{p}}\left\{x^{T}(k) Q_{i} x(k)+u^{T}(k) R_{i} u(k)+x^{T}(k+1) P_{k+1}^{*} x(k+1)\right\} \\
= & \min _{i \in M, u(k) \in R^{p}}\left\{x^{T}(k) Q_{i} x(k)+u^{T}(k) R_{i} u(k)+\left[A_{i} x(k)+B_{i} u(k)\right]^{T} P_{k+1}^{*}\left[A_{i} x(k)+B_{i} u(k)\right]\right\} \\
= & \min _{i \in M, u(k) \in R^{p}}\left\{x^{T}(k)\left[Q_{i}+A_{i}^{T} P_{k+1}^{*} A_{i}\right] x(k)+u^{T}(k)\left[R_{i}+B_{i}^{T} P_{k+1}^{*} B_{i}\right] u(k)\right. \\
& \left.\quad+2 x^{T}(k) A_{i}^{T} P_{k+1}^{*} B_{i} u(k)\right\} .
\end{aligned}
$$

Let

$$
S_{i}(u)=u^{T}\left(R_{i}+B_{i}^{T} P_{k+1}^{*} B_{i}\right) u+2 x^{T}(k) A_{i}^{T} P_{k+1}^{*} B_{i} u
$$

By simple calculation, we have

$$
\frac{\partial S_{i}(u)}{\partial u}=2\left(R_{i}+B_{i}^{T} P_{k+1}^{*} B_{i}\right) u+2 B_{i}^{T} P_{k+1}^{*} A_{i} x(k) .
$$

Since $u(k)$ is unconstrained, its optimal value $u_{i}^{*}(k)$ must satisfy $\partial S_{i}(u) / \partial u=0$.

It follows that

$$
u_{i}^{*}(k)=-\left(R_{i}+B_{i}^{T} P_{k+1}^{*} B_{i}\right)^{-1} B_{i}^{T} P_{k+1}^{*} A_{i} x(k)=-K_{i}\left(P_{k+1}^{*}\right) x(k)
$$


It follows that

$$
\begin{aligned}
& J_{2, k}(u, r)= \min _{i \in M, u(k) \in R^{p}}\left\{x^{T}(k)\left[Q_{i}+A_{i}^{T} P_{k+1}^{*} A_{i}\right] x(k)+u_{i}^{* T}(k)\left[R_{i}+B_{i}^{T} P_{k+1}^{*} B_{i}\right] u_{i}^{*}(k)\right. \\
&\left.\quad+2 x^{T}(k) A_{i}^{T} P_{k+1}^{*} B_{i} u_{i}^{*}(k)\right\} \\
&= \min _{i \in M}\left\{x^{T}(k)\left[Q_{i}+A_{i}^{T} P_{k+1}^{*} A_{i}\right] x(k)+x^{T}(k) K_{i}^{T}\left(P_{k+1}^{*}\right)\left[R_{i}+B_{i}^{T} P_{k+1}^{*} B_{i}\right] K_{i}\left(P_{k+1}^{*}\right) x(k)\right. \\
&\left.\quad-2 x^{T}(k) A_{i}^{T} P_{k+1}^{*} B_{i} K_{i}\left(P_{k+1}^{*}\right) x(k)\right\} \\
&=\min _{i \in M, P \in L_{k+1}} x^{T}(k) f_{i}(P) x(k) \\
&=\min _{P \in L_{k}} x^{T}(k) P x(k) .
\end{aligned}
$$

Then the optimal switching signal and the optimal control input at time $k$ are $\gamma^{*}(k)=i_{k}^{*}$ and $u^{*}(k)=-K_{i_{k}^{*}}\left(P_{k}^{*}\right) x(k)$, respectively. It means that (3.31) still holds for $k$. This completes the proof.

\section{Examples}

Example 4.1. Let us consider the following discrete-time switched linear system:

$$
x(k+1)=A_{\sigma(k)} x(k)+B_{\sigma(k)} u(k), \quad k=0,1, \ldots, N-1, \sigma(k) \in M=\{1,2\},
$$

where

$$
A_{1}=\operatorname{diag}(-1,-2), \quad A_{2}=\operatorname{diag}(10,10), \quad B_{1}=\left[\begin{array}{l}
1 \\
1
\end{array}\right], \quad B_{1}=\left[\begin{array}{l}
1 \\
2
\end{array}\right]
$$

The parameters in simulations are as follows:

$$
Q_{1}=\operatorname{diag}(0.1,0.1), \quad Q_{2}=(0.2,0.2), \quad R_{1}=1, \quad R_{2}=0.1, \quad Q_{f}=\operatorname{diag}(1,1), \quad N=400 .
$$

We design the controllers with the approach in Theorem 3.1, at the initial state $x_{0}=\left[\begin{array}{ll}1 & -1\end{array}\right]^{T}$ of the system; the state response of closed-loop discrete-time switched linear system is as in Figure 1.

Example 4.2. Let us consider the following discrete-time switched linear system borrowed from [32]:

$$
x(k+1)=A_{\sigma(k)} x(k)+B_{\sigma(k)} u(k), \quad k=0,1, \ldots, N-1, \sigma(k) \in M=\{1,2\},
$$




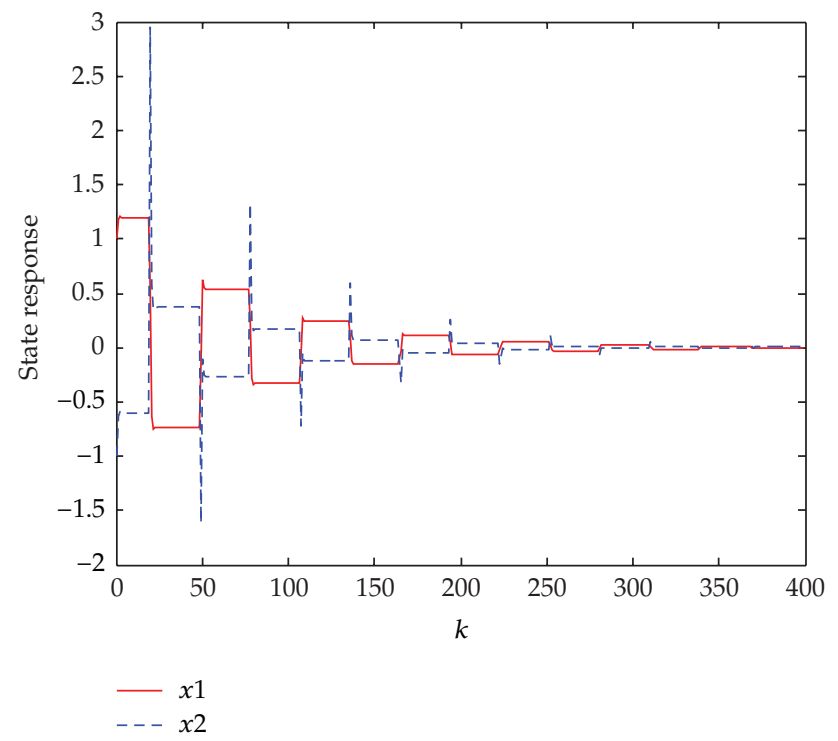

Figure 1: The state response of closed-loop system.

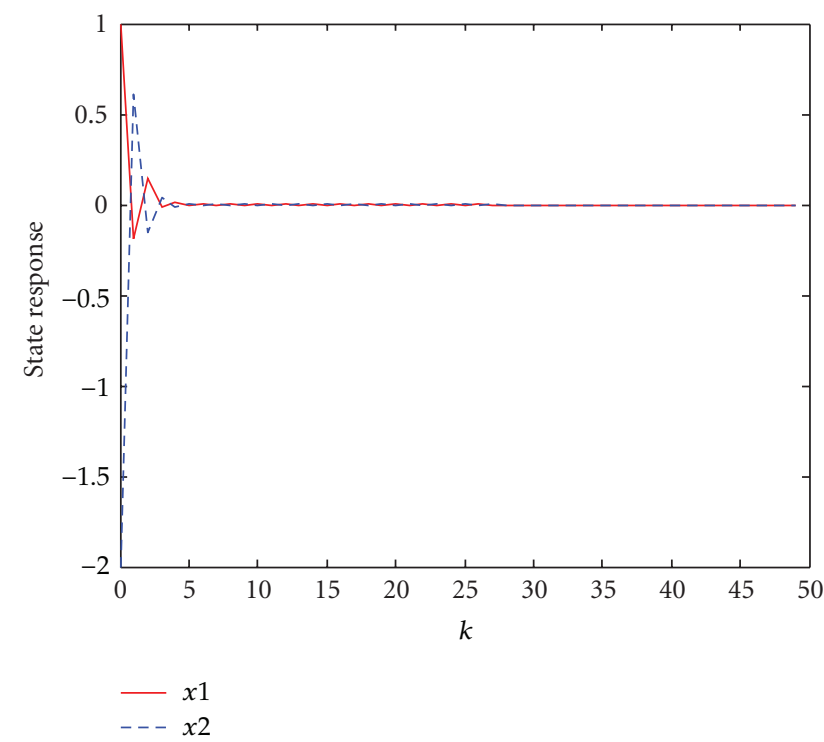

Figure 2: The state response of closed-loop system.

where

$$
A_{1}=\left[\begin{array}{ll}
0.545 & -0.430 \\
0.185 & -0.610
\end{array}\right], \quad A_{2}=\left[\begin{array}{cc}
-0.555 & -0.37 \\
0.215 & -0.590
\end{array}\right], \quad B_{1}=\left[\begin{array}{c}
1 \\
0.5
\end{array}\right], \quad B_{1}=\left[\begin{array}{l}
1 \\
3
\end{array}\right]
$$


The parameters in simulations are as follows:

$$
Q_{1}=\operatorname{diag}(1,1), \quad Q_{2}=\operatorname{diag}(2,2), \quad R_{1}=0.1, \quad R_{2}=0.1, \quad Q_{f}=\operatorname{diag}(10,10), \quad N=50 .
$$

We design the controller in with the approach in Theorem 3.1, at the initial state $x_{0}=\left[\begin{array}{ll}1-2\end{array}\right]^{T}$ of the system; the closed-loop state response of discrete-time switched linear system is as in Figure 2.

\section{Conclusions}

Based on dynamic programming, finite-horizon optimal quadratic regulations are studied for discrete-time switched linear systems. The finite-horizon optimal quadratic control strategies minimizing the cost function are given for discrete-time switched linear systems, including optimal continuous controller and discrete-time controller. The infinite-horizon optimal quadratic regulations of discrete-time switched linear system will be investigated in the future.

\section{Acknowledgments}

The authors wish to acknowledge the reviewer for his comments and suggestions, which are invaluable for significant improvements of the readability and quality of the paper. The authors would like to acknowledge the National Nature Science Foundation of China for its support under Grant no. 60964004, 60736022, 61164013 and 61164014, China Postdoctoral Science Foundation for its support under Grant no. 20100480131, Young Scientist Raise Object Foundation of Jiangxi Province, China for its support under Grant no. 2010DQ01700, and Science and Technology Support Project Plan of Jiangxi Province, China for its support under Grant no. 2010BGB00607.

\section{References}

[1] J. Ezzine and A. H. Haddad, "Controllability and observability of hybrid systems," International Journal of Control, vol. 49, no. 6, pp. 2045-2055, 1989.

[2] M. S. Branicky, "Multiple Lyapunov functions and other analysis tools for switched and hybrid systems," IEEE Transactions on Automatic Control, vol. 43, no. 4, pp. 475-482, 1998.

[3] D. Cheng, "Controllability of switched bilinear systems," IEEE Transactions on Automatic Control, vol. 50, no. 4, pp. 511-515, 2005.

[4] D. Cheng, L. Guo, Y. Lin, and Y. Wang, "Stabilization of switched linear systems," IEEE Transactions on Automatic Control, vol. 50, no. 5, pp. 661-666, 2005.

[5] D. Cheng, Y. Lin, and Y. Wang, "Accessibility of switched linear systems," IEEE Transactions on Automatic Control, vol. 51, no. 9, pp. 1486-1491, 2006.

[6] S. S. Ge, Z. Sun, and T. H. Lee, "Reachability and controllability of switched linear discrete-time systems," IEEE Transactions on Automatic Control, vol. 46, no. 9, pp. 1437-1441, 2001.

[7] Z. Sun, "Canonical forms of switched linear control systems," in Proceedings of the American Control Conference (AAC '04), pp. 5182-5187, Boston, Mass, USA, July 2004.

[8] Z. Sun, S. S. Ge, and T. H. Lee, "Controllability and reachability criteria for switched linear systems," Automatica, vol. 38, no. 5, pp. 775-786, 2002.

[9] Z. Sun and S. Sam Ge, "Dynamic output feedback stabilization of a class of switched linear systems," IEEE Transactions on Circuits and Systems I, vol. 50, no. 8, pp. 1111-1115, 2003. 
[10] Z. Sun and S. S. Ge, "Analysis and synthesis of switched linear control systems," Automatica, vol. 41, no. 9, pp. 181-195, 2005.

[11] Z. Sun and S. S. Ge, "Switched stabilization of higher-order switched linear systems," in Proceedings of the 44th IEEE Conference on Decision and Control, and the European Control Conference (CDC-ECC '05), pp. 4873-4878, Seville, Spain, December 2005.

[12] Z. Sun and S.S. Ge, Switched linear systems, Control and Design Series: Communications and Control Engineering, Springer, New York, NY, USA, 2005.

[13] Z. Sun and D. Zheng, "On reachability and stabilization of switched linear systems," IEEE Transactions on Automatic Control, vol. 46, no. 2, pp. 291-295, 2001.

[14] X. M. Sun, J. Zhao, and D. J. Hill, "Stability and $\mathrm{L}_{2}$-gain analysis for switched delay systems: a delaydependent method," Automatica, vol. 42, no. 10, pp. 1769-1774, 2006.

[15] Y. G. Sun, L. Wang, and G. Xie, "Necessary and sufficient conditions for stabilization of discretetime planar switched systems," Nonlinear Analysis, Theory, Methods and Applications, vol. 65, no. 5, pp. 1039-1049, 2006.

[16] Y. G. Sun, L. Wang, G. Xie, and M. Yu, "Improved overshoot estimation in pole placements and its application in observer-based stabilization for switched systems," IEEE Transactions on Automatic Control, vol. 51, no. 12, pp. 1962-1966, 2006.

[17] G. Xie and L. Wang, "Necessary and sufficient conditions for controllability of switched linear systems," in Proceedings of the American Control Conference, pp. 1897-1902, May 2002.

[18] G. Xie and L. Wang, "Reachability realization and stabilizability of switched linear discrete-time systems," Journal of Mathematical Analysis and Applications, vol. 280, no. 2, pp. 209-220, 2003.

[19] G. Xie and L. Wang, "Controllability and stabilizability of switched linear-systems," Systems and Control Letters, vol. 48, no. 2, pp. 135-155, 2003.

[20] J. Zhao and D. J. Hill, "Dissipativity theory for switched systems," IEEE Transactions on Automatic Control, vol. 53, no. 4, pp. 941-953, 2008.

[21] J. Zhao and D. J. Hill, "Passivity and stability of switched systems: a multiple storage function method," Systems and Control Letters, vol. 57, no. 2, pp. 158-164, 2008.

[22] Z. Ji, X. Guo, L. Wang, and G. Xie, "Robust Ho control and stabilization of uncertain switched linear systems: a multiple lyapunov functions approach," Journal of Dynamic Systems Measurement and Control, vol. 128, no. 3, pp. 696-700, 2006.

[23] Z. Ji, L. Wang, and X. Guo, "Design of switching sequences for controllability realization of switched linear systems," Automatica, vol. 43, no. 4, pp. 662-668, 2007.

[24] Z. Ji, G. Feng, and X. Guo, "A constructive approach to reachability realization of discrete-time switched linear systems," Systems and Control Letters, vol. 56, no. 11-12, pp. 669-677, 2007.

[25] M. Johansson and A. Rantzer, "Computation of piecewise quadratic Lyapunov functions for hybrid systems," IEEE Transactions on Automatic Control, vol. 43, no. 4, pp. 555-559, 1998.

[26] D. Liberzon and A. S. Morse, "Basic problems in stability and design of switched systems," IEEE Control Systems Magazine, vol. 19, no. 5, pp. 59-70, 1999.

[27] W. Zhang, A. Abate, J. Hu, and M. P. Vitus, "Exponential stabilization of discrete-time switched linear systems," Automatica, vol. 45, no. 11, pp. 2526-2536, 2009.

[28] R. A. Decarlo, M. S. Branicky, S. Pettersson, and B. Lennartson, "Perspectives and results on the stability and stabilizability of hybrid systems," Proceedings of the IEEE, vol. 88, no. 7, pp. 1069-1082, 2000.

[29] X. Xu and P. Anstraklis, "A dynamic programming approach for optimal control of switched systems," IEEE Transactions on Automatic Control, vol. 49, no. 1, pp. 2-16, 2004.

[30] M. Egerstedt, Y. Wardi, and F. Delmotle, "Transition-time optimization for switched-mode dynamical systems," IEEE Transactions on Automatic Control, vol. 5, no. 1, pp. 1110-1115, 2004.

[31] W. Zhang and J. Hu, "On optimal quadratic regulation for discrete-time switched linear systems," in Proceedings of the 11th International Conference on Hybrid Systems: Computation and Control, pp. 584-597, April 2008.

[32] B. Hu, X. Xu, P. J. Antsaklis, and A. N. Michel, "Robust stabilizing control laws for a class of secondorder switched systems," Systems and Control Letters, vol. 38, no. 3, pp. 197-207, 1999. 


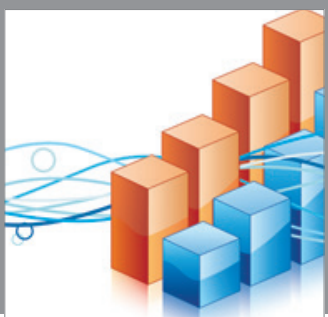

Advances in

Operations Research

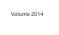

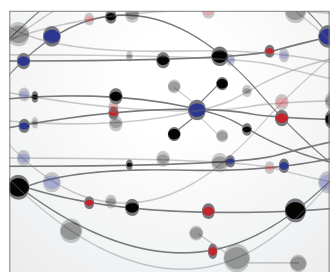

\section{The Scientific} World Journal
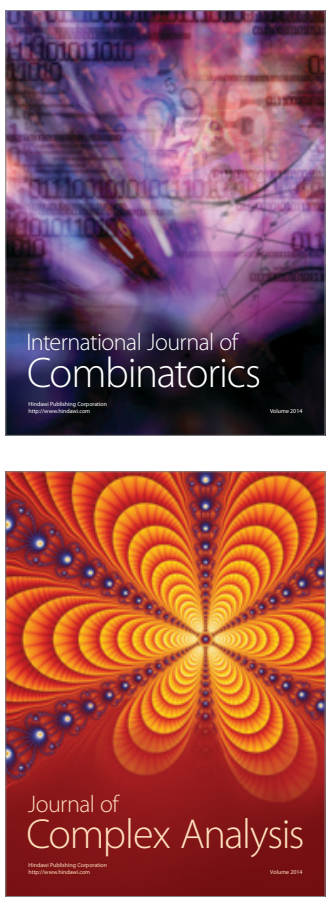

International Journal of

Mathematics and

Mathematical

Sciences
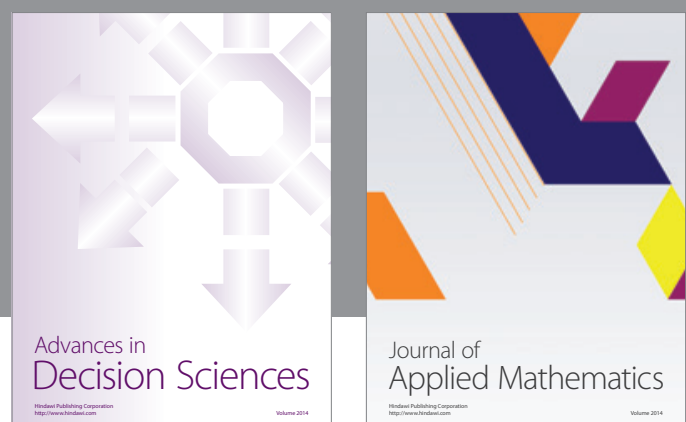

Journal of

Applied Mathematics
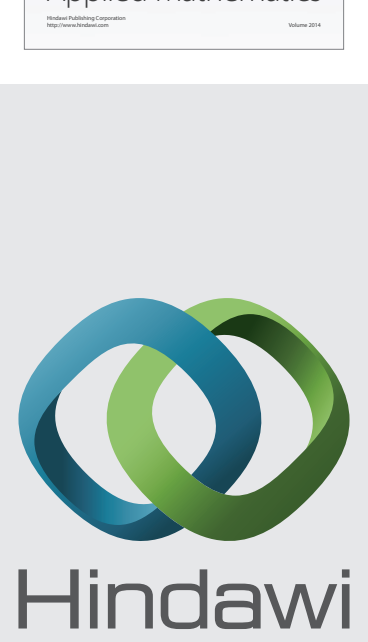

Submit your manuscripts at http://www.hindawi.com
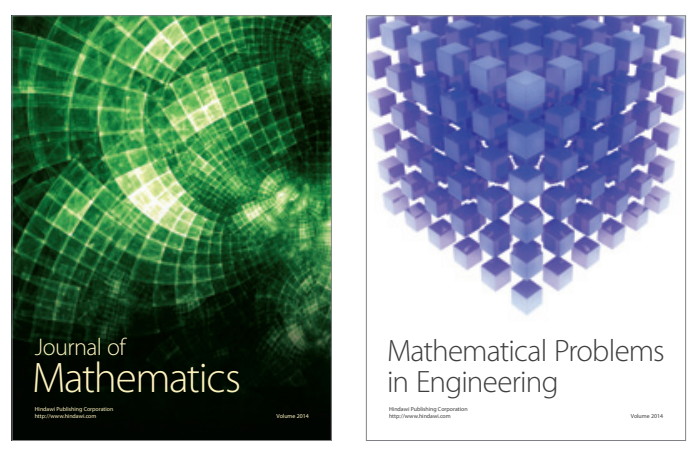

Mathematical Problems in Engineering
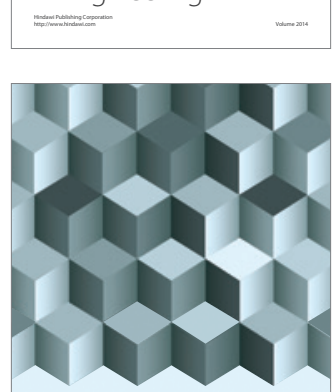

Journal of

Function Spaces
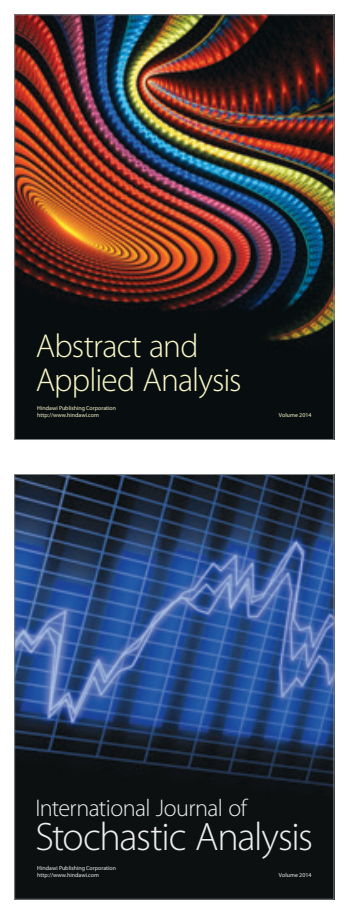

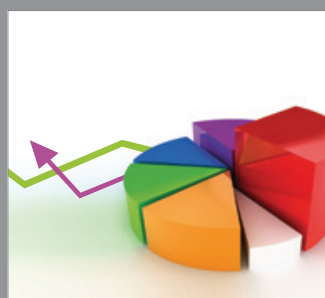

ournal of

Probability and Statistics

Promensencen
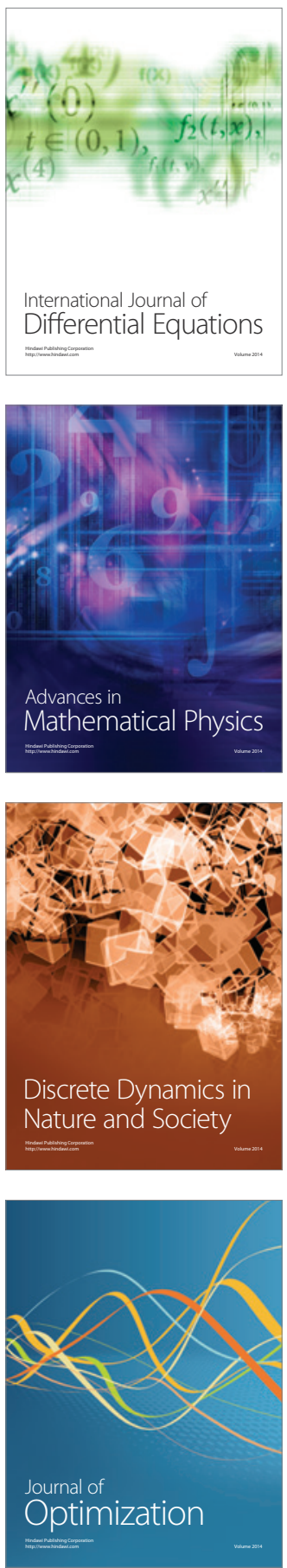